\author{
N. CHAOUI ${ }^{1, \infty, *}$ \\ J. SOLIS ${ }^{1}$ \\ C.N. AFONSO ${ }^{1}$ \\ T. FOURRIER ${ }^{2}$ \\ T. MUEHLBERGER ${ }^{2}$ \\ G. SCHREMS ${ }^{2}$ \\ M. MOSBACHER ${ }^{2}$ \\ D. BÄUERLE ${ }^{2}$ \\ M. BERTSCH ${ }^{3}$ \\ P. LEIDERER ${ }^{3}$
}

\section{A high-sensitivity in situ optical diagnostic technique for laser cleaning of transparent substrates}

\author{
${ }^{1}$ Instituto de Optica, CSIC, c/ Serrano 12, 28006 Madrid, Spain \\ ${ }^{2}$ Institut für Experimentalphysik, J. Kepler Universität, Altenberger Str. 69, 4040 Linz, Austria \\ ${ }^{3}$ Fachbereich Physik und Optikzentrum, Fach M676, Universität Konstanz, 78457 Konstanz, Germany
}

\begin{abstract}
A differential optical transmission technique has been used to monitor in situ the efficiency of laser cleaning for the removal of sub-micrometer-sized particles on substrates transparent at the monitoring wavelength. This technique has been applied to the removal of sub-micrometer polystyrene particles on polyimide substrates using laser pulses of $30 \mathrm{ps}$ duration at $292 \mathrm{~nm}$ while probing the material transmission at $633 \mathrm{~nm}$. The sensitivity achieved $-1 / 10^{4}$ for the transmission changes induced upon single-pulse laser exposure - allows us to monitor the removal of just a few sub-micron-sized particles from the probed region inside the irradiated area.
\end{abstract}

PACS 81.65.Cf

1

\section{Introduction}

Laser cleaning [1-10] appears as one of the most promising techniques for the removal of sub-micron-sized particles from surfaces. The strong adhesion of these contaminants to the surface makes conventional processes inefficient, this problem being of major importance, among others, in the field of microelectronics. Two different procedures for laser cleaning have been described in the literature: dry laser cleaning (DLC) [1-5] and steam laser cleaning (SLC) [1,6-10]. DLC is based on the interaction between the incident laser light and the substrate to be cleaned. So far, it has been assumed that the thermal expansion, as a consequence of light absorption, was the unique mechanism responsible for particle removal [1]. However, it has very recently been shown that local field enhancement effects [11-13] underneath the particles can lead to local ablation of the substrate. This effect is undesirable for the particle-removal process. SLC differs from DLC in the fact that a thin liquid film (water/alcohol mixture) is deposited on the sample by steam condensation, prior to irradiation. The mechanism responsible for particle ejection in SLC is believed to be the explosive evaporation

Fax: +33-3/87844082, E-mail: nchaoui@iut.univ-metz.fr

* Present address: Laboratoire de Chimie et Applications, Institut Universitaire de Technologie de Metz, Département Chimie, Rue Victor Demange, 57500 Saint-Avold, France of the liquid film in the vicinity of the particle/substrate interface [1].

The cleaning efficiency is normally defined as $\left(1-n / n_{0}\right)$, where $n_{0}$ and $n$ are respectively the density of particles on the surface before and after laser exposure. This parameter is usually determined by time-consuming post-treatment examination of the samples by means of microscopy techniques. The need for making the use of laser cleaning compatible with online monitoring tools for industrial applications has led to the development of in situ diagnostic methods for laser cleaning, often based on optical measurements. For instance, scattered light reflection measurements have successfully been applied to quantitatively monitor the removal of sub-micron particles from silicon wafers upon both SLC and DLC $[8,9]$. The linear dependence observed between the intensity of scattered light at the surface and the areal density of scattering particles [2] allows us to perform a direct correlation between the changes in the amount of the scattered light and the efficiency of the particle-removal process. The use of optical techniques for such a purpose is not new, as commercial systems for unpatterned surface inspection also often use optical techniques based on bright- and dark-field illumination as well as scattered light detection in order to monitor surface defects and contaminants. These techniques are normally combined with the use of electron beams for both imaging and the assessment of electrical defects.

Nevertheless, optical methods for surface inspection based on scattered light measurements require a smooth and homogeneous surface and a relatively large reflectivity at the monitoring wavelength in order to obtain a significant optical contrast between contaminated and cleaned (or defectfree) regions. The increasing importance of the use of some "transparent materials" like some spin-on-glasses or polymers [14] in the field of microelectronics makes it necessary to develop and to optimize surface-cleaning techniques for removing sub-micrometer contaminants from their surfaces. However these materials, unlike silicon, are normally nearly transparent to visible radiation, which makes the use of conventional scattered reflectivity measurements for monitoring surface particle densities to become of little use or at least complicated. In addition, in some other cases, like conventional industrial polymers, the material surface is rough. There is therefore a need for techniques that can provide a reliable in situ monitoring of the laser cleaning process, despite the 
surface roughness and/or the weak specular reflectivity of the substrate.

In this report, we describe an in situ and non-invasive diagnostic technique that allows measuring the very small transmission changes induced over the size of the probe beam by the removal of a few sub-micrometer-sized particles from a substrate transparent at the monitoring wavelength. As a case study, we present results obtained in laser cleaning experiments using UV picosecond (ps) pulses to remove polystyrene spheres dispersed on the surface of polyimide substrates.

\section{$2 \quad$ Experimental details 2.1 Sample preparation}

Mono-disperse polystyrene (PS) spheres deposited on the surface of polyimide (PI) substrates were used as model contaminants. The particle diameters used in these experiments were 320 and $800 \mathrm{~nm}$. Details of the procedure for sample preparation can be found elsewhere [2]. Briefly, the particles, available in an isopropyl alcohol colloidal suspension, were deposited onto $250 \mu \mathrm{m}$ thick PI foils (Goodfellow) by means of spin coating. Figure 1 shows a representative scanning electron microscopy (SEM) image of a PI foil covered with $800 \mathrm{~nm}$ sized PS spheres. After evaporation of the solvent, even when some aggregates can be observed, the particles are mostly isolated and statistically well distributed over the surface. The surface density of particles before laser treatment $\left(n_{0}\right)$ is about $1.5 \times 10^{4} \mathrm{~mm}^{-2}$. This initial areal density of particles, well above the contamination which would be considered a routine level in the semiconductor industry, was chosen in order to make a clear determination of the sensitivity of the differential transmission technique for measuring "in situ" the efficiency of the laser cleaning process.

\section{2}

\section{Cleaning and diagnostic set-up}

The experimental set-up used to remove the particles and to monitor the process is shown in Fig. 2. It es-

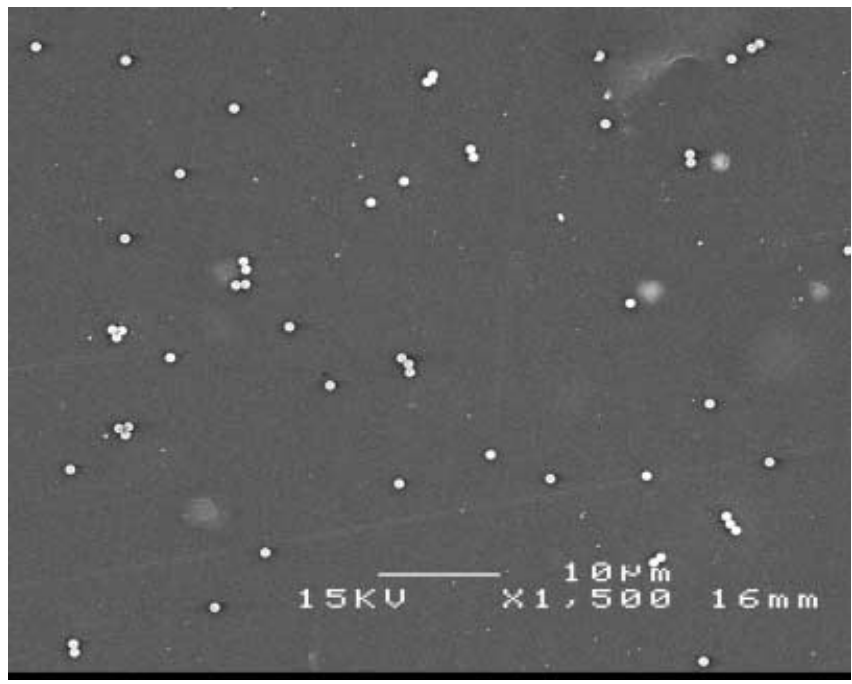

FIGURE 1 SEM image of a typical sample before laser cleaning showing a random distribution of mainly isolated polystyrene spheres with a diameter of $800 \mathrm{~nm}$

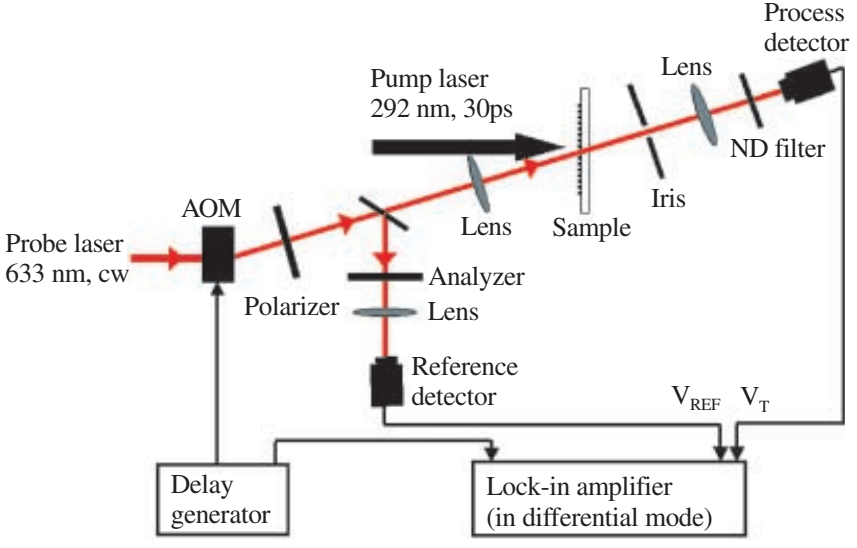

FIGURE 2 Experiment layout showing the pump beam $(\lambda=292 \mathrm{~nm}, \tau=$ 30 ps FWHM) that irradiates the sample surface and the cw probe laser $(\lambda=$ $633 \mathrm{~nm})$ used to monitor the transmission change $(\Delta T)$ upon laser exposure (see text for more details)

sentially consists of a pump beam that irradiates the sample surface in a single shot and a cw probe laser used to monitor the transmission change $(\Delta T)$ upon laser exposure. The sample is located in a translation stage in order to expose a fresh region of the surface to the pump pulse in each single-pulse irradiation. Since the aim of the study was to determine the sensitivity of the "in situ" transmission measurements to the changes of the areal density of particles after laser exposure, the possible re-deposition of particles was not impeded by any means like the use of an inert-gas flow.

The irradiation laser system is based on a synchronously pumped tunable dye laser that provides pulses with a duration of $10 \mathrm{ps}$ (FWHM). The output of this laser (tuned at $\lambda=584 \mathrm{~nm}$ ) is amplified by a dye amplifier pumped by a frequency-doubled Q-switched Nd:YAG laser. The amplified beam ( $30 \mathrm{ps}$ FWHM) is then frequency-doubled $(\lambda=$ $292 \mathrm{~nm}$ ) by means of a beta barium borate (BBO) crystal and focused at normal incidence onto the sample surface. The spatial intensity distribution of the laser spot at the sample site is Gaussian elliptical with 1/e dimensions of $\sim 180 \mu \mathrm{m}$ (long axis) and $\sim 90 \mu \mathrm{m}$ (short axis).

The probe beam is delivered by a He-Ne laser $(633 \mathrm{~nm})$. Its amplitude is modulated by means of an acousto-optic modulator $(\mathrm{AOM})$ with a square-wave signal at a repetition rate of $1071 \mathrm{~Hz}$. The He-Ne laser beam passes through a polarizer and a beam splitter (10\% reflectivity). The reflected part of the beam is then sent to a reference photodiode detector after passing through an analyzer, while the transmitted part is focused with a lens to a spot size of $\sim 30 \mu \mathrm{m}$ (1/e radius) at the center of the irradiated region at an angle of incidence of $15^{\circ}$. The intensity of the beam transmitted through the sample is measured by means of another photodiode. Both reference and transmission detectors are similar and are connected to a lock-in amplifier in differential mode.

The use of an analyzer in front of the reference diode allows us to cancel the difference between the voltage readings of the reference $\left(V_{\mathrm{REF}}\right)$ and transmission $\left(V_{\mathrm{T}}\right)$ detectors, before exposing the fresh surface to the irradiation pulse. After exposure, the differential signal $V_{\mathrm{T}}-V_{\mathrm{REF}}$ is obviously related to the transmission changes caused by the removal of the particles and/or the possible modification of the substrate 
induced by the irradiation beam. With the photodiodes and polarizing elements used we have been able to resolve transmission changes of the order of $1 / 10^{4}$. Post-treatment examination of the samples has been performed by means of optical microscopy, SEM and atomic force microscopy (AFM) in order to correlate the transmission changes measured with the state of the surface.

3

\section{Results}

Figure 3 shows the evolution of the differential transmission changes induced upon single-pulse exposure for surfaces covered with PS spheres ( 800 and $320 \mathrm{~nm}$ diameter) as a function of the laser fluence. The plot shows the existence of a threshold fluence, $F_{\mathrm{th}} \approx 6 \mathrm{~mJ} / \mathrm{cm}^{2}$, above which transmission changes of the order of $2 / 10^{3}(0.2 \%)$, well above the experimental resolution, are observed for both particle sizes. Above this threshold, both curves exhibit a similar evolution that can be divided into three different fluence regimes. For regime I $\left(6 \mathrm{~mJ} / \mathrm{cm}^{2}<F<10 \mathrm{~mJ} / \mathrm{cm}^{2}\right)$, the signal increases sharply as the fluence is increased until it reaches a plateau. The plateau, regime II, is observed for fluences $10 \mathrm{~mJ} / \mathrm{cm}^{2} \leq F<22 \mathrm{~mJ} / \mathrm{cm}^{2}$. The transmission change in regime II is slightly higher for the films covered with $800 \mathrm{~nm}$ particles $(\Delta V \approx 0.1 \mathrm{~V}$, equivalent to $a \approx 1 \%$ transmission change) than for those covered with $320 \mathrm{~nm}$ particles $(\Delta V \approx$ $0.07 \mathrm{~V}$, equivalent to $a \approx 0.7 \%$ transmission change). Above $F=22 \mathrm{~mJ} / \mathrm{cm}^{2}$, regime III, the transmission increases again with fluence upon laser exposure for both particle sizes.

Figure 4 is an optical micrograph showing two columns of spots irradiated with several different fluences in a specimen covered with $800 \mathrm{~nm}$ PS particles. In order to enhance the contrast between laser-exposed regions and the surroundings, grazing-incidence illumination with an intense whitelight source was used. The lower brightness observed at the irradiated spots in these "dark-field" images is clearly related to the decrease of scattering associated with the removal of the particles, especially considering the large initial density of PS spheres on the surface. Images obtained after irradiation at

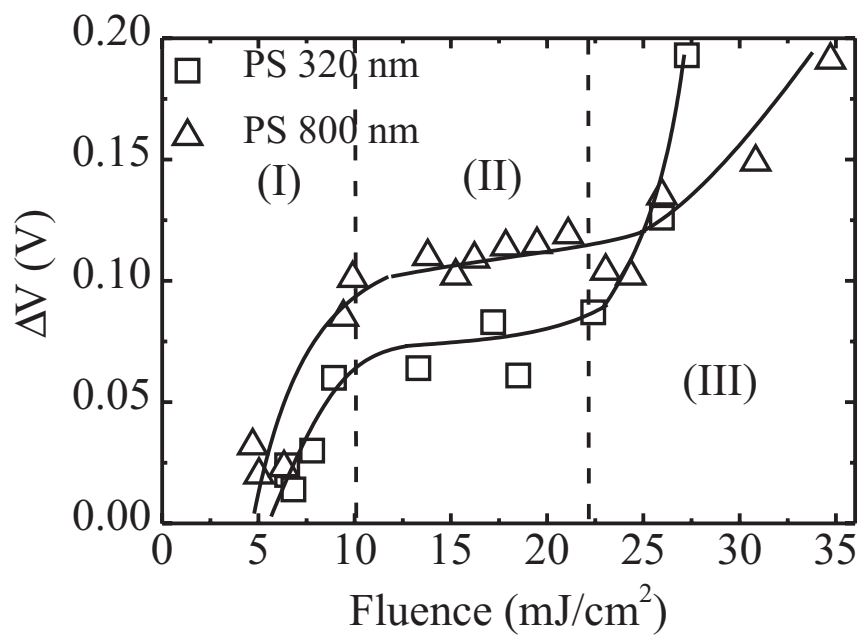

FIGURE 3 Differential transmission change after exposure to a single laser pulse as a function of fluence for PS spheres of $800 \mathrm{~nm}(\triangle)$ and $320 \mathrm{~nm}(\square)$ diameter on a PI substrate

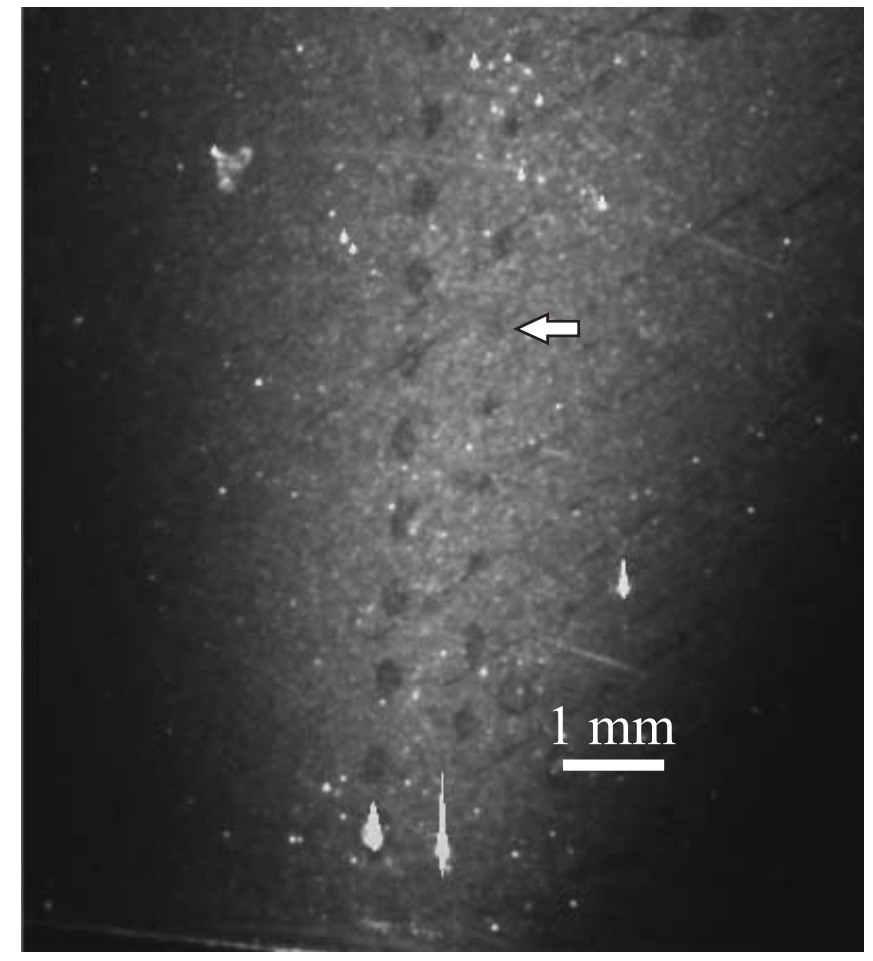

FIGURE 4 Optical micrograph of a PI sample with 800-nm size particles showing two strings of irradiated spots at fluences within regime III (left row of spots) and within regimes I and II (right row of spots). The arrow points to a spot irradiated at the cleaning threshold fluence

similar fluences in substrates not initially covered by particles do not show any observable contrast. The spots in the left column, exposed to a single pulse with a constant fluence within regime III, exhibit an approximately constant size. The spots in the right column, irradiated with single pulses of increasing fluence within regimes I and II, show an increase of size as we move downwards. The arrow points at a spot irradiated with a fluence at the transmission-change threshold.

The correlation between the transmission changes and the removal of the particles was further analyzed by means of SEM. Figure 5 shows an illustrative image of a region irradiated with a fluence at the edge between regimes I and II for a sample initially covered with $800 \mathrm{~nm}$ sized PS particles and irradiated at a fluence of $10 \mathrm{~mJ} / \mathrm{cm}^{2}$. It can be seen that for this fluence all the particles have been removed, thus indicating that already for this fluence a $100 \%$ local cleaning efficiency has been achieved. A closer inspection of the treated surface (higher-magnification inset in Fig. 5) shows however that at the positions previously occupied by the particles (marked by arrows in the figure), the material surface is locally deformed or ablated. This effect is observed in the SEM images for all the fluences above the transmissionchange threshold $\left(6 \mathrm{~mJ} / \mathrm{cm}^{2}\right)$, further confirming the relation of the transmission changes and the areal density of particles.

In order to further analyze the correlation of the measured transmission changes with the state of the surface, additional analysis of the substrate after laser exposure was performed by AFM in irradiated regions not initially covered by particles. Power spectral density (PSD) curves in the $0.2-1 \mu \mathrm{m}$ range were calculated from AFM micrographs recorded at the 


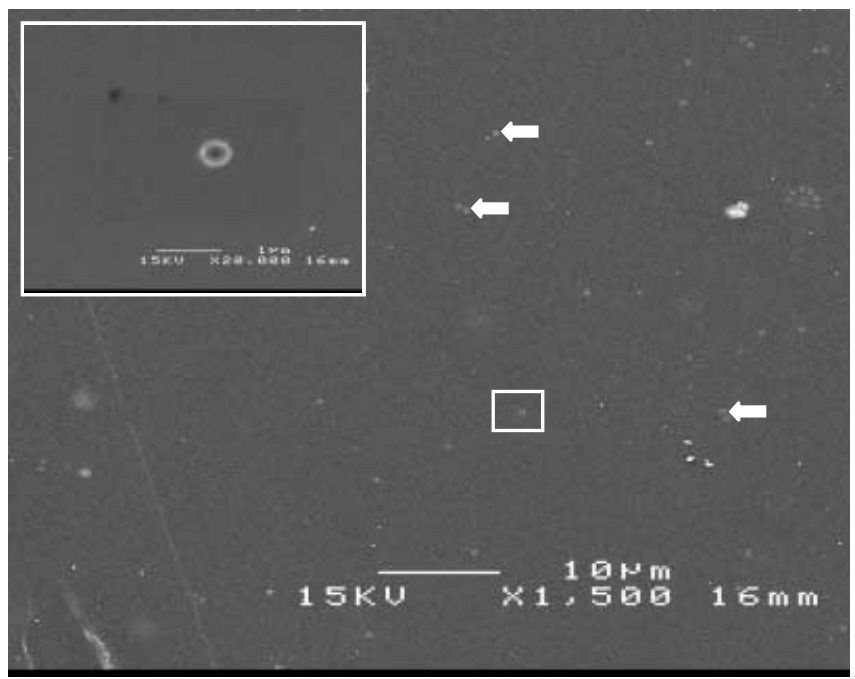

FIGURE 5 SEM image of a cleaned area of a sample of PI formerly covered with 800-nm PS particles (see Fig. 1 for comparison). The region was irradiated with a fluence within regime II. The white arrows indicate damage pits underneath the former locations of the particles. One of these damage pits is shown with higher magnification in the inset

central part of the irradiated spots. The PSD curves provide information about the roughness spatial frequencies correlated to light scattering: at a randomly rough surface, many different spatial frequencies are present. The PSD curves provide the relative strength of each roughness component of the surface microstructure as a function of spatial frequency. Figure 6 shows PSD curves corresponding to regions irradiated at fluence regimes II and III together with that corresponding to a non-irradiated region. For both regimes, the surface roughness increases upon laser exposure. However, when the laser fluence is increased from regime II to regime III, a slight decrease of the PSD is observed all over the range corresponding to visible-light wavelengths. Thus, at the probe wavelength $(\lambda=633 \mathrm{~nm})$, the surface irradiated within regime III scatters slightly less light than upon irradiation within regime II.

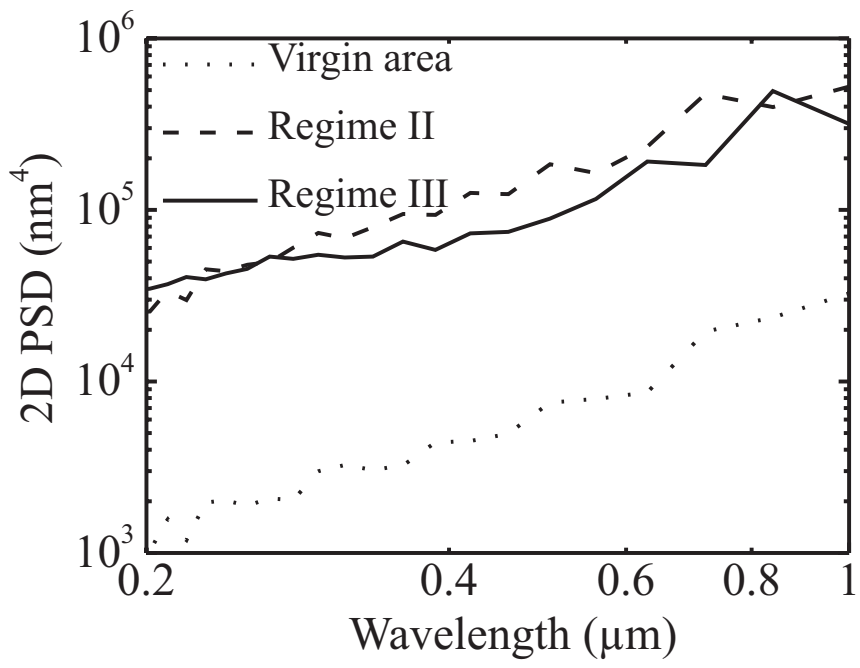

FIGURE 6 2D power spectral density measured at the central part of two representative spots irradiated with fluences within regime II, regime III as well as at a non-exposed area

\section{4}

\section{Discussion}

The obvious decrease of scattering observed in the laser irradiated-regions (Fig. 4) and its comparison with the transmission changes (Fig. 3) and SEM images (Fig. 5) clearly indicates that, within fluence regimes I and II, the transmission changes induced upon laser exposure are essentially related to the removal of particles from the surface. Since for a fluence of $10 \mathrm{~mJ} / \mathrm{cm}^{2}$ a $100 \%$ cleaning efficiency is already achieved (Fig. 5), the plateau in Fig. 3 (regime II) corresponds to a situation in which the transmission change associated with the removal of the particles from the substrate does saturate. In other words, a cleaning efficiency of $100 \%$ is induced over a region with a size clearly higher than that of the probe beam independently of the laser fluence used. As a consequence, the transmission changes observed in regime I for increasing fluences must be caused either by the fluence dependence of the cleaning mechanism or by the increasing size of the cleaned region when compared to the size of the probe beam. Figure 4 clearly indicates that the latter explanation is most likely since the size of the cleaned areas within regime I increases with fluence and is smaller than the size of the probe beam, something which has been confirmed by SEM examination of different regions within a cleaned spot below and above the threshold fluence for detecting changes in transmission.

The different plateau levels observed for $320 \mathrm{~nm}$ and $800 \mathrm{~nm}$ particle sizes in regime II are therefore due to the different sizes of the particles, which gives rise to different amounts of transmission change upon complete removal of the particles from the surface. In both cases, considering that the initial density of particles is about $1.5 \times 10^{4} \mathrm{~mm}^{-2}$ and the size of the probed region is about $30 \mu \mathrm{m}$ (1/e radius), it can be concluded that the reflectivity change at the plateau in Fig. 3 corresponds to the removal of just some tens of particles from the probed region. An extrapolation of this value allows us to see that the transmission change recorded at the cleaning threshold fluence would correspond to the removal of just a few particles from the surface, which is an indication of the extreme sensitivity achieved. In this sense it is important to bear in mind that the fact that the technique used is differential makes its sensitivity to be independent of the initial density of particles. In other words, the detection limit for a change in the number of particles in the probed region after laser exposure is just a few particles no matter the initial particle density, which makes this technique compatible with its use under initial contamination levels many orders of magnitude smaller than the ones used in this case study.

The global picture of the fluence dependence of the transmission changes within regimes I and II given in the previous paragraphs is further supported by the comparison of the PSD curves (Fig. 6) corresponding to regime II and to a non-treated region. This figure shows that the irradiated surface is rougher than the non-treated one all over the spectral interval analyzed. If the measured changes in the roughness of the surface were to cause any transmission change, the observed transmission changes would be negative (transmission decrease) and not positive (transmission increase) as we observe all over the studied fluence interval. This figure also indicates that the deformation/ablation features remaining at the former loca- 
tion of the particles (see the inset of Fig. 5) do not affect significantly the transmitted light intensity, as their effect would be, if any, to increase and not to decrease the amount of scattering.

The deformation/ablation of the surface at the former location of the laser-removed particles could be considered as an indication of local ablation of the substrate being the removal mechanism. This mechanism would be driven by local field enhancement [11-13] underneath the particles. In certain cases, this field enhancement can lead to fluences 10 times higher than the nominal average laser fluence used. As a consequence, it is not difficult to reach the damage threshold of the substrate, especially for the high peak powers associated with the ps laser pulses used in the present work for cleaning. Therefore, the cleaning efficiency should be a bimodal function of the fluence (no removal below the local ablation threshold or $100 \%$ efficiency above), in agreement with the features observed in Figs. 3 and 4 for fluences within regime I, in which the transmission increase (Fig. 3) appears essentially caused by the increasing size of the cleaned area (Fig. 4) and not by an increase of the cleaning efficiency. This would also be consistent the SEM observation of regions irradiated at fluences below and above threshold $\left(6 \mathrm{~mJ} / \mathrm{cm}^{2}\right)$. In any case other processes, like particle explosion or surface shrinkage under the particles, could potentially contribute to the removal mechanism and to the features observed in the surface after laser exposure.

Finally, when the fluence is further increased (regime III), even when the particle-removal efficiency has already reached $100 \%$, the transmission of the sample increases again upon exposure. This transmission increase can not be justified by the small decrease of the surface roughness observed for visible optical frequencies when the fluence reaches regime III (see Fig. 6), since we have already seen that much stronger changes in the surface roughness (Fig. 6, comparison of nontreated sample with treated one in regime II) give rise to no measurable transmission changes $\left(\Delta T / T_{0}<10^{-4}\right)$ in regions not initially covered by particles. As a consequence, the observed transmission increase observed for increasing fluences within regime III in Fig. 3 must be associated with a modification of the optical constants of the PI substrate at high fluences, although this latter conclusion requires further investigation. This as well as the determination of the actual removal mechanism of the PS particles on PI upon pulsed UV laser exposure is beyond the scope of this paper, as this system has been only used for a case study in order to assess the actual sensitivity of differential trans- mission measurements to monitor the efficiency of the laser cleaning process in substrates transparent at the monitoring wavelength.

We have seen that the technique is sensitive to the removal of just a few particles from the probed region of a transparent substrate, which makes it of clear potential interest for the assessment of laser cleaning procedures in microelectronics and optical industry applications. In the former case, even if the transparent material is spin-coated or deposited on a nontransparent substrate, the possibility of using a transparent substrate alongside the actual manufactured material would make its use of interest.

\section{$5 \quad$ Conclusion}

We have shown that differential transmission measurements provide a powerful tool to monitor in situ the efficiency of laser cleaning of sub-micrometer particles on transparent rough substrates. The application of this technique to PI films covered with PS spheres cleaned by UV ps laser pulses has allowed us to detect the transmission changes associated with the removal of just a few particles from the probed region at the threshold fluence for cleaning.

ACKNOWLEDGEMENTS Support of this work by the European Union in the framework of the TMR project Laser Cleaning (Contract No. ERB-FMRXCT-980188) is gratefully acknowledged.

\section{REFERENCES}

1 A.C. Tam, W.P. Leung, W. Zapka, W. Ziemlich: J. Appl. Phys. 71, 3515 (1992)

2 T. Fourrier, G. Schrems, T. Mühlberger, J. Heitz, N. Arnold, D. Bäuerle, M. Mosbacher, J. Boneberg, P. Leiderer: Appl. Phys. A 72, 1 (2001)

3 G. Vereecke, E. Röhr, M.M. Heyns: J. Appl. Phys. 85, 3837 (1999)

4 Y.F. Lu, Y.W. Zheng, W.D. Song: J. Appl. Phys. 87, 1534 (2000)

5 D.R. Halfpenny, D.M. Kane: J. Appl. Phys. 86, 6641 (1999)

6 K. Imen, J. Lee, S.D. Allen: Appl. Phys. Lett. 58, 203 (1991)

7 M. She, D. Kim, C.P. Grigopoulos: J. Appl. Phys. 86, 6519 (1999)

8 M. Mosbacher, N. Chaoui, J. Siegel, V. Dobler, J. Solis, J. Boneberg, C.N. Afonso, P. Leiderer: Appl. Phys. A 69, 331 (1999)

9 M. Mosbacher, V. Dobler, J. Boneberg, P. Leiderer: Appl. Phys. A 70, 669 (2000)

10 X. Wu, E. Sacher, M. Meunier: J. Appl. Phys. 87, 3618 (2000)

11 P. Leiderer, J. Boneberg, V. Dobler, M. Mosbacher, H.-J. Münzer, N. Chaoui, J. Siegel, J. Solis, C.N. Afonso, T. Fourrier, G. Schrems, D. Bäuerle: Proc. SPIE 4065, 249 (2000)

12 M. Mosbacher, H.-J. Münzer, J. Zimmermann, J. Solis, J. Boneberg, P. Leiderer: Appl. Phys. A 72, 41 (2001)

13 B.S. Luk'yanchuk, Y.W. Zheng, Y.F. Lu: Proc. SPIE 4423, 115 (2001)

14 P.K.H. Ho, D.S. Thomas, R.H. Friend, N. Tessler: Science 285, 233 (1999) 